\title{
Energy relaxation in molecular systems containing salt ions
}

\author{
M. A. Baranov ${ }^{1}$, E. N. Velichko ${ }^{1}$, E. K. Nepomnyashchaya ${ }^{1}$, I. V. Pleshakov ${ }^{2}$ \\ ${ }^{1}$ Peter the Great St. Petersburg Polytechnic University, \\ Polytechnicheskaya, 29, Saint Petersburg, 195251, Russia \\ ${ }^{2}$ Ioffe Institute, 26 Politechnicheskaya str., Saint Petersburg, 194021, Russia \\ baranovma1993@gmail.com, velichko-spbstu@yandex.ru, elina.nep@gmail.com, ivanple@yandex.ru
}

PACS 05.40.-a, 05.65.+b, 02.70.Ns, 87.14.Ee, 87.15.He

DOI 10.17586/2220-8054-2021-12-5-598-602

\begin{abstract}
In this paper, we consider relaxation processes in molecular systems containing single biomolecule and salt ions. The energy fluctuations in such systems were evaluated using computer simulations. The comparative analysis of the free energy dynamics of alanine, tryptophan, and albumin biomolecules in constructed molecular systems (aqueous solutions with different degrees of ionization) resulted in high influence of ionizing impurities on the full energy of the system and on the energy relaxation time. The results obtained can be used for development of hybrid micro and nanoelectronic devices with built-in biomolecular objects, for example, biochemical sensors, devices with microflow of liquids, technology for the preparation of molecular films, etc.
\end{abstract}

Keywords: self-organization, proteins, molecular dynamics, biomolecular electronics, energy relaxation.

Received: 1 October 2021

Revised: 16 October 2021

\section{Introduction}

Like in the living systems, the characteristics of hybrid micro and nanoelectronic devices with organic molecules are determined by specific realizations of the structure, dynamics and function of biomolecules and their ensembles [1]. The Key influence on the biomolecular properties of the proteins and peptides, is determined by biomolecular conformation, energy, and dipole moment. These parameters of the molecules in ionized solutions are required for development of highly-efficient modern bioelectronic devices, such as biochemical sensors. The computer modeling is to be used to investigate biomolecules, and in particular, to determine the probability of establishing a certain conformation [2-5], since conformational changes are correlated with changes in the dipole moment and with total energy of molecule or molecular cluster [6-9].

In this study we use the molecular modeling method to investigate changes of the molecular conformation and of the ionization degree of their clusters during the relaxation processes.

\section{Calculation methods}

Visual Molecular Dynamics software was utilized to develop molecular system models of different structure. This method is usually used for a computer modelling of the free energy of large molecules [2,10,11]. The model of the molecular system was presented by one biomolecule placed in a water cube. In all considered systems, the distance from the molecule to the cube faces (box padding) was equal to $15 \AA$.

In this study, we used albumin, glycine, and tryptophan molecules the modelling. In considering the dissolution of the molecules, the TIP3W model of water was used. This model assumes three atoms of a water at three points of interaction. To create salt solutions with concentration $\mathrm{n}[\mathrm{mol} / \mathrm{L}]$ in the water cube, the standard VMD function of adding ions was used. Changing of the concentration of ions was achieved by introduction of the $\mathrm{NaCl}$ ions with a minimum distance of $5 \AA$. Similar methods are also utilized in other published experiments [12-14]. The addition of $\mathrm{Na}^{+}$and $\mathrm{Cl}^{-}$ions was used to change salt concentration in the interval from $1.71 \times 10^{-4}$ to $1.71 \mathrm{~mol} / \mathrm{L}$, which corresponds to $0.001-10 \%$. The maximum concentration of ions in this case was about 3200 ions per molecule in the water cube.

All simulations were performed with a CHARMM27 force field using the NAMD package with the periodic boundary conditions and with the cutoff radius for unrelated interactions with a switching function, starting at $9 \AA$ and reaching zero at $12 \AA$ for the aqueous medium. To establish equilibrium in the system, the energy was minimized and the conformational state of the molecules was stabilized for 50 ps. As a result, the stable conformational states 
of molecules were obtained. For the Energy minimization and following calculation of the molecular relaxation, the Langevin equation was used:

$$
m_{i} \frac{d^{2} x_{i}(t)}{d t^{2}}=F_{i}\left(x_{i}(t)\right)-\gamma_{i} \frac{d x_{i}(t)}{d t^{2}} m_{i}+R_{i}(t),
$$

here, $\gamma_{i} m_{i}$ is the system damping factor, $\gamma_{i}$ is a friction coefficient, $m_{i}$ is the particle mass, $F_{i}$ stands for the force acting on the particle by the electric field (for example, ions), and $R_{i}$ is the random force that acts on the particle. The Langevin method enables calculation of the kinetic energy of the system while simultaneously controlling the temperature of the molecular object and the pressure. A change in the concentration of ions in the solution leads to a change in the relaxation and the stabilization times.

Calculation of the energy value of the molecular cluster in an aqueous solution was based on the Free Energy Perturbation algorithm in the Nanoscale Molecular Dynamics program. The algorithm consists in evaluation of the energy difference between two states of the system at each moment of time that allows prediction of the system dynamic scenario. Since the calculations were performed for a constant pressure, the free energy is associated with the Gibbs energy [15].

Simulation of the relaxation begins immediately after energy minimization. The relaxation step was analyzed for 150 ps using an NPT ensemble. The instantaneous pressure in the water cube was presented by the root mean square fluctuations of $\frac{k T}{V \beta}$ value, where $\beta$ is compressibility. The pressure was obtained by spatio-temporal averaging of the kinetic pressure $\frac{n R T}{V}$ and the compensating negative static pressure.

As a result, the values of the total energy of molecular clusters were obtained depending on the simulation time. The total energy can be represented as a sum of the kinetic and the potential energies (Eqs. 2,3):

$$
\begin{gathered}
H(p, r)=K(p)+U(r), \\
K(p)=\sum_{i=1}^{N} \frac{p_{i}^{2}}{2 m_{i}} .
\end{gathered}
$$

The potential energy is presented by the sum of various potential energies, describing the bound and the unbound interactions between the particles (Eq. 4):

$$
U_{\text {total }}=U_{\text {bond }}+U_{\text {angle }}+U_{\text {dihedral }}+U_{v d W}+U_{\text {Coulomb }}
$$

The first three terms describe the stretching, bending, and torsional bonded interactions, where the bonds count includes each covalent bond in the system, angles are the angles between each pair of covalent bonds sharing a single atom at the vertex, and dihedral describes atom pairs separated by exactly three covalent bonds with the central bond subject to the torsion angle. The final two terms in eq. (4) describe interactions between nonbonded atom pairs: van der Waal's forces and electrostatic interactions.

Based on these values, the expected experimental dependences of the relaxation energies on time and on the concentration of salt ions in solutions were obtained and analyzed.

\section{Results and discussion}

As a result of our calculations, the dependences of the total energy on the simulation time were obtained. Fig. 1 shows typical time dependences of full energy of the molecular clusters for the albumin, glycine, and tryptophan with concentration of $\mathrm{NaCl}$ salt ions equal to $0.15 \mathrm{~mol} / \mathrm{L}$. It is assumed that oscillations of the molecular cluster occur at the relaxation site, when the energy from the protein molecules is transferred to the aqueous environment. Fig. 1 shows that the larger the molecule, the less time it takes for its oscillation energy to relax. The relaxation time of molecular system was determined from the values of the energies of molecular system when the amplitude of vibrations of the total energy of molecules became less than $10 \%$ of the maximum value.

Fig. 2 shows the dependence of the relaxation energy (that corresponds to the relaxation time) on the salt concentration in the molecular cluster. The salt concentration is presented in dimensionless values and was calculated as the decimal logarithm of the ratio of the salt concentration to the initial salt concentration.

The energy value established as a result of relaxation is proportional to the number of molecules in the water cube and the mass of the biomolecule under study. The total energy $E$ also depends on the concentration of ions and decreases upon stabilization by an amount of $\Delta \mathrm{E}$, which is much smaller than $\mathrm{E}$. The rate of the energy decline depends on the size of the molecule that for glycine consists of 3202 atoms, for tryptophan of 4131 atoms and for albumin has 169169 atoms . This means that in the process of relaxation, only a small part of the initial total energy is lost. Most likely, this part refers to kinetic energy. In Fig. 3 kinetic energy of molecular system is presented. One 

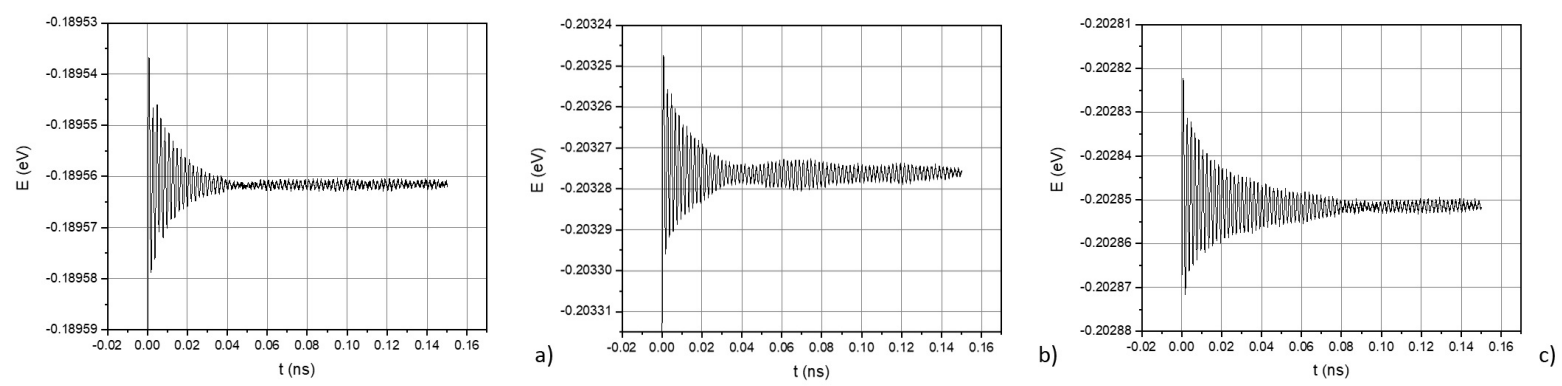

FIG. 1. Relaxation of molecular cluster energy shown as a function of time for a) albumin molecule b) glycine molecule c) tryptophane molecule

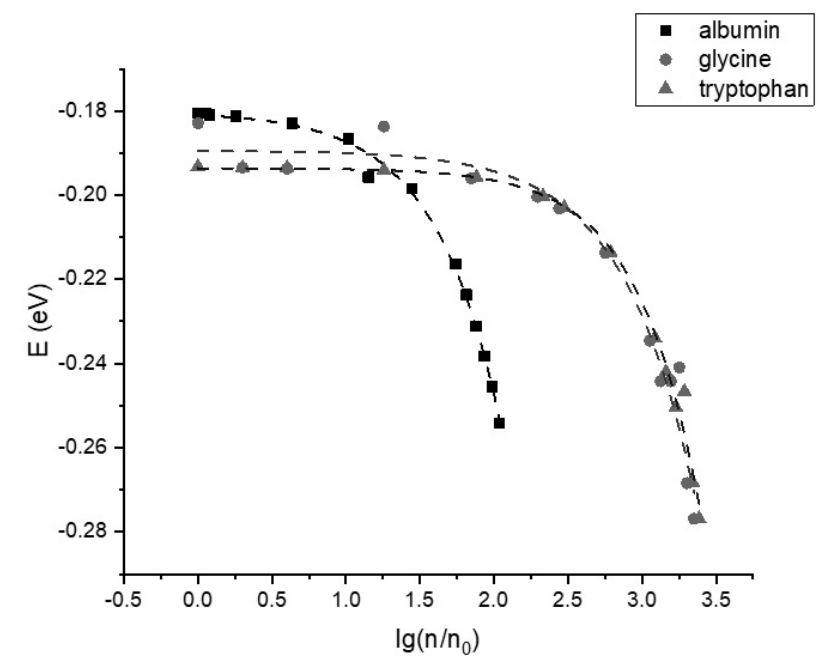

FIG. 2. Relaxation energy of tryptophan, albumin and glycine molecules shown as function of $\mathrm{n}(\mathrm{NaCl})$

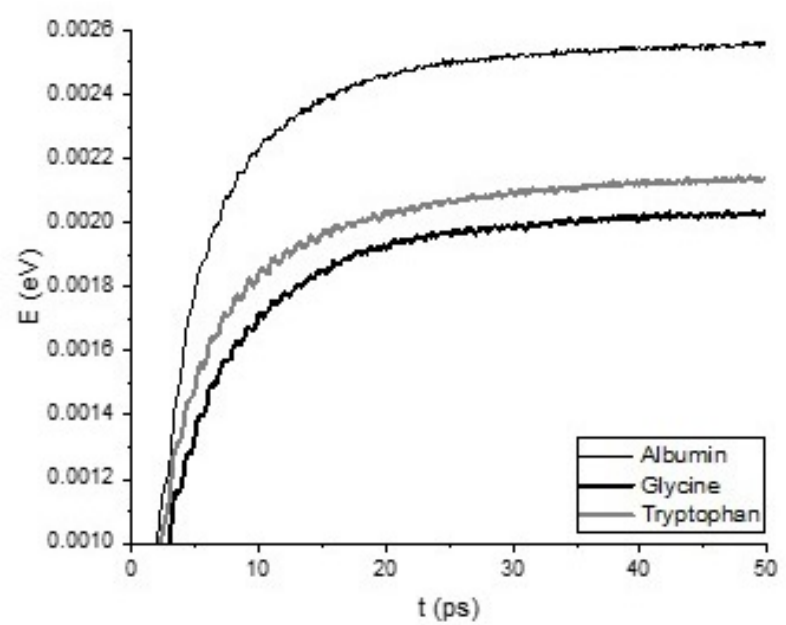

FIG. 3. Kinetic energy of tryptophan, albumin and glycine molecules shown as function of time 


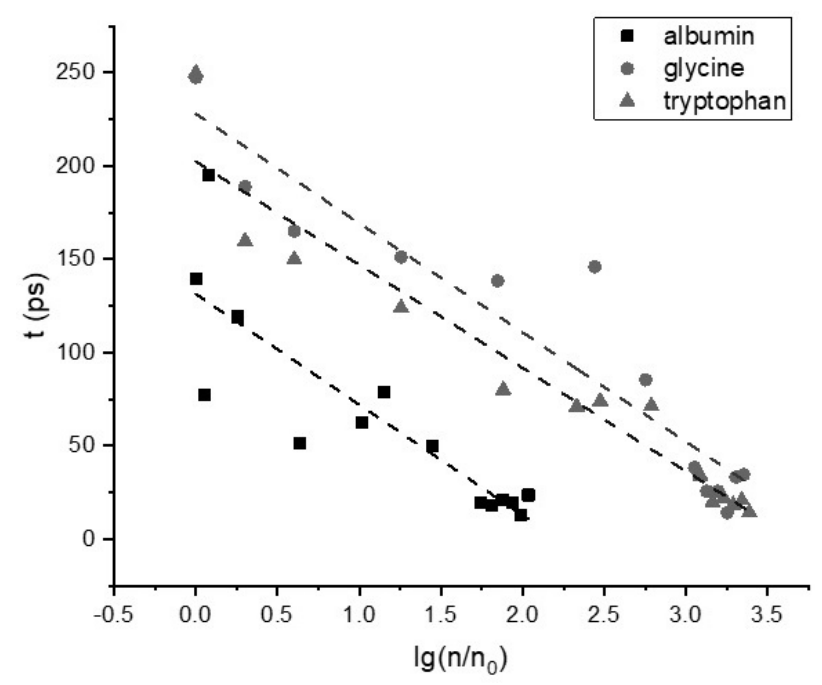

FIG. 4. Relaxation time of tryptophan, albumin and glycine molecules shown as function of $\mathrm{n}(\mathrm{NaCl})$

can notice, that in the process of relaxation of the total energy of a molecular cluster, the energy is transferred from the protein to ions. As a result, there is an increase in the kinetic energy.

The characteristic time of energy relaxation was also investigated for the studied molecular systems. Fig. 4 shows the dependences of the relaxation times on the logarithm of the salt ion concentration in the molecular system. As can be seen from Fig. 3, with an increase of the salt concentration, the relaxation time of the energy in studied molecular systems decreases. Analyzing these dependences, the smaller molecules reach the saturation level more slowly at the same ion concentration. However, the larger molecules require more ions to achieve equilibrium.

As can be seen from our modeling, presented in this section, the total energy and energy relaxation time depend on the type of protein and the number of atoms in the system. The energy relaxation process is caused by the initial nonequilibrium state of the molecular cluster with respect to the solution. As a result, we observe energy flux from the cluster to the solution.

\section{Conclusion}

Summarizing, in our research, we have applied the molecular simulation method to study relaxation processes in molecular systems. It was shown that the initial free energy of a molecular cluster decreases spontaneously during the dissipation time, when the cluster energy is mainly lost to the solution. It reaches the quasi-stationary level when the equilibrium of energy fluxes from the cluster to the solution and back is established.

The relaxation energy of the solution is transferred into the ionic component, or in other words, into the ion vibrations during the salt dissociation. The relaxation energy proves to be much smaller than the total energy of the system. Therefore, we can conclude that the kinetic energy is mainly transferred during these processes.

The characteristic time of the energy reduction to the stable state varies with the salt concentration. As the salt concentration in the solvation cube increases, the relaxation time decreases. In addition, the free energy of a molecular cluster also decreases with an increase in the salt concentration in the solution. This corresponds to a further decrease in the electric field at the cluster surface due to an increase in the $\mathrm{pH}$ and electrical conductivity of the aqueous saline solution.

Energy relaxation that occurs with slow aperiodic oscillations corresponds to relaxation of a dissipative dynamic system with low electrical conductivity and increased "friction". Apparently, this corresponds to an electric field at the cluster surface created by polar water molecules and dissolved salt ions.

The results provided by this study can be useful for the development of modern biomolecular electronic elements constructed from single molecules and molecular layers.

\section{Acknowledgement}

This research was funded by RSF, grant number No 21-72-20029. The results were obtained using computational resources of Peter the Great Saint Petersburg Polytechnic University Supercomputing Center (www.spbstu.ru).

The Authors express their deep gratitude to Prof. O. Yu. Tsybin for many fruitful discussions and invaluable advices which have helped us to plan the experiments and explain experimental data. 


\section{References}

[1] Zotti L.A. Molecular Electronics. Applied Sciences, 2021, 11, P. 4828.

[2] Wang Y., Harrison C.B., Schulten K., McCammon J.A. Implementation of accelerated molecular dynamics in NAMD. Comput. Sci. Discov., 2011, P. 4.

[3] Viloria J.S., Allega M.F., Lambrughi M., Papaleo E. An optimal distance cutoff for contact-based Protein Structure Networks using side-chain centers of mass. Sci. Rep., 2017, 7, P 1-11.

[4] Petty M.C., Nagase T., Suzuki H., Naito H. Molecular electronics. Springer Handbooks, $2017,1$.

[5] Hasani H.J., Ganesan A., Ahmed M., Barakat K.H. Effects of protein-protein interactions and ligand binding on the ion permeation in KCNQ1 potassium channel. PLoS One, 2018, 13.

[6] Baranov M., Tsybin O. and Velichko E. Structured biomolecular films for microelectronics. Saint Petersbg. Polytech. State Univ.Journal. Phys. Math., 2021, 14, P. 85-99.

[7] Bibi F., Villain M., Guillaume C., Sorli B., Gontard N.A. Review: Origins of the Dielectric Properties of Proteins and Potential Development as Bio-Sensors. Sensors, 2016, 16(8), P. 1232.

[8] Taylor P.A., Jayaraman A. Molecular Modeling and Simulations of Peptide-Polymer Conjugates Annu. Rev. Chem. Biomol. Eng., 2020, 11, P. 257-76.

[9] Xiao B.L., Ning Y.N., Niu N.N., Li D., Moosavi-Movahedi A.A., Sheibani N., Hong J. Steered molecular dynamic simulations of conformational lock of $\mathrm{Cu}, \mathrm{Zn}$-superoxide dismutase. Sci. Rep., 2019, 9, P. 1-11.

[10] Tavanti F., Pedone A., Menziani M.C. A closer look into the ubiquitin corona on gold nanoparticles by computational studies. New J. Chem., 2015, 39, P. 2474-82.

[11] Tavanti F., Pedone A. and Menziani M. C. Competitive Binding of Proteins to Gold Nanoparticles Disclosed by Molecular Dynamics Simulations J. Phys. Chem. C, 2015, 119, 22172-80

[12] Baranov M., Velichko E., Rozov S. Dehydrated films of protein solutions: structural properties. Saint Petersbg. Polytech. State Univ. Journal. Phys. Math-ematics, 2019, 12, P. 25-37.

[13] Dyubo D., Tsybin O.Y., Baranov M.A., Alekseenko A.P., Velichko E.N. Study of electric properties of self-assembled films of albumin during their dehydration Recent citations Study of electric properties of self-assembled films of albumin during their dehydration. J. Phys, 2018, 1124, P. 31013.

[14] Phillips J.C., Braun R., Wang W., Gumbart J., Tajkhorshid E., Villa E., Chipot C., Skeel R. D., Kalé L., Schulten K. Scalable molecular dynamics with NAMD. J. Comput. Chem., 2005, 26, P. 1781-802.

[15] Sanchez C., Galo G.J., Ribot F. and Grosso D. Design of functional nano-structured materials through the use of controlled hybrid organicinorganic interfaces. Comptes Rendus Chim., 2003, 6, P. 1131-51. 